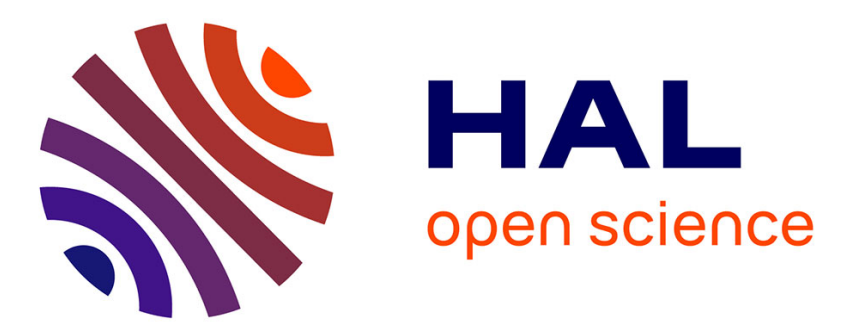

\title{
Attentional limits in visual search with and without dorsal parietal dysfunction: space-based window or object-based span?
}

Audrey Vialatte, Romeo Salemme, Aarlenne Zein Khan, Laure Pisella

\section{To cite this version:}

Audrey Vialatte, Romeo Salemme, Aarlenne Zein Khan, Laure Pisella. Attentional limits in visual search with and without dorsal parietal dysfunction: space-based window or object-based span? Neuropsychologia, 2021, 161, pp.108013. 10.1016/j.neuropsychologia.2021.108013 . hal-03366458

\section{HAL Id: hal-03366458 \\ https://hal.science/hal-03366458}

Submitted on 5 Oct 2021

HAL is a multi-disciplinary open access archive for the deposit and dissemination of scientific research documents, whether they are published or not. The documents may come from teaching and research institutions in France or abroad, or from public or private research centers.
L'archive ouverte pluridisciplinaire HAL, est destinée au dépôt et à la diffusion de documents scientifiques de niveau recherche, publiés ou non, émanant des établissements d'enseignement et de recherche français ou étrangers, des laboratoires publics ou privés. 
Attentional limits in visual search with and without dorsal parietal dysfunction: space-based window or object- based span?

Vialatte Audrey ${ }^{1}$, Salemme Romeo ${ }^{1}$, Khan Aarlenne Zein ${ }^{1,2}$, Pisella Laure ${ }^{1}$

1 Lyon neuroscience Research Center (CRNL), Trajectoires Team, INSERM U1028, CNRS UMR 5292, Université Claude Bernard, Lyon, France

2 School of Optometry, University of Montreal, Montreal, Canada

Address correspondence to L. Pisella PhD, 16 avenue du doyen Lépine, 69676 Bron, France. Email: laure.pisella@inserm.fr.

Highlights

- For identical symbols, a limited space-based attentional window is used.

- For dissimilar symbols, attention is based on a limited object-based span.

- SPL damage reduces the spatial attentional window for identical symbols.

- SPL damage drastically reduces the object number span for dissimilar symbols. 


\section{Abstract}

Attentional resource and distribution are specifically impaired in simultanagnosia, and also in the visuo-attentional form of developmental dyslexia. Both clinical conditions are conceived as a limitation of simultaneous visual processing after superior parietal lobule (SPL) dysfunction (review in Valdois et al. 2019). However, a reduced space-based attentional window (i.e. a limited visual eccentricity at which the target object can be identified, Khan et al. 2016) has been demonstrated in simultanagnosia versus a reduced object-based span (i.e. a limited number of objects processed at each fixation, Bosse et al. 2007) in developmental dyslexia. In healthy individuals, the cost in reaction times per item in serial search tasks suggests that a group of objects is processed simultaneously at a time, but this group is also undefined and depends on task visual complexity.

Healthy individuals and a patient with simultanagnosia performed serial search tasks involving either symbols (made of separable features) or objects made of non-separable features, and with distractors that were either all identical or all dissimilar. We used a gaze-contingent moving window paradigm to determine whether the task was performed with a "working space" versus a "working span" limitation in control group and in patient with bilateral SPL damage.

We found that healthy individuals performed search in a color task comprising nonseparable feature objects and dissimilar distractors with a limited space-based attentional window; this attentional window, as well as the mean saccade amplitude used to displace it across the visual display, were independent of set size, thus inconsistent with an object-based attentional span. In the symbol task comprising a feature-absent search in which all feature-present distractors were dissimilar, we observed that mean saccade amplitude decreased with set size and that search performance could not be mimicked by a gaze-contingent visible window of a single diameter; instead participants seemed to process a fixed number of symbols at a time (object-based span).

Following bilateral SPL lesions, patient IG demonstrated a similar space-based search process in the color search task with a normal attentional window. In contrast, her costper-item in the symbol task increased dramatically, demonstrating a clear deficit of simultaneous object perception. These results confirmed the specific contribution of the SPL to the visual processing of multiple objects made of separable features (like letters), and more dramatically when they are all different, which explains the specific difficulty for a reading beginner in case of SPL dysfunction.

Key words:

visual perception, posterior parietal cortex, simultaneous processing, visual search, simultanagnosia, developmental dyslexia 


\section{INTRODUCTION}

Bálint (1909, and later Michel and Henaff, 2004) described a shrunk functional "field of view" in a patient who manifested no attention for visual events appearing outside central vision, having neither a visual field deficit nor oculomotor paralysis. This component of the Balint's triad consecutive to bilateral damage to the superior parietal lobule (SPL) is now often considered a deficit of objects' simultaneous perception called simultanagnosia (a term coined by Wolpert, 1924). The original report of Balint (1909) already reflected the remaining ambiguity between a space-based versus an object-based attentional limitation consecutive to bilateral posterior parietal damage: it described "an extreme restriction of visual attention, such that only one object is seen at a time" (Bálint, 1909; Husain and Stein, 1988).

With a similar ambiguity, in healthy individuals performing a serial search task there is evidence that changing the set size (i.e. number of distractors) does not change reaction times as would be expected by an object by object account of search (Khan et al., 2016; Young and Hulleman, 2013). Instead, it suggests that that a given group of stimuli is processed together for each task. A recent study (Khan et al., 2016) yield two main results. First, visual search in healthy individuals was performed with a limited spatial attentional workspace specific to a given task difficulty, as previously suggested by Young and Hulleman (2013); all objects within this workspace are processed in one fixation, since gaze-contingent visible window of a given diameter exhibited a search time cost corresponding to a fixed off-set, irrespective of the number of distractors falling within this window (independent of set size). Second, simultanagnosia was a shrinking of this workspace consecutive to bilateral SPL lesions, as previously suggested by Michel and Henaff (2004), but only for stimuli made of separable features; patient IG was significantly slower in feature-present and feature-absent visual searches (comprising objects with and without an additional lines) and not in tasks involving objects without any separable feature (searches of a single feature or requiring conjunction of shape and color features). The gaze-contingent moving window paradigm demonstrated that this slowness was due to a reduced attentional "working space", i.e. a smaller attentional distribution around fixation. Patient IG's mean search time in full view was comparable to the controls' mean 
search time when they performed the pop-out balloon search task with a gaze-contingent visible window of $15^{\circ}$, independent of set size. Moreover, performing this task with a gazecontingent visible window of $20^{\circ}$ did not produce any cost in search time to find the target among 12, 24 or 48 distractors for this patient IG (Khan et al., 2016).

Interestingly, similar specific visual search deficits for objects made from separable features have been reported in poor readers. Casco and Prunetti (1996) tested several visual search displays of various difficulties in poor and good readers. Poor readers were not slower to detect targets when they were distinguished by a single feature of color or orientation, or by a conjunction of non-separable features (combination of orientation and color, or of orientation and shape). They were slower when the target or the distractors involved separable features, regardless of whether these stimuli were non-verbal symbols or letters. It has also been demonstrated that several patients with developmental dyslexia show a visuo-attentional (VA) span deficit, in isolation or in association to a phonological deficit, both contributing independently to poor reading skills (Bosse et al., 2007; Germano et al., 2014; Zoubrinetzky et al., 2016, 2014). Behaviorally, this VA span deficit has been highlighted using the Global Report task in which a multi-consonant string is briefly and centrally presented on a computer screen and participants are required to orally report all the consonants. The string is presented for $200 \mathrm{~ms}$ to avoid being processed longer than one ocular fixation. Dyslexics can only process a reduced (compared to controls) number of distinct consonants simultaneously (in one ocular fixation) due to limited visual attention capacity (Bogon et al., 2014; Lobier et al., 2013). The reduced span of report in dyslexics is not specific to letter strings since it is also observed for digits and non-verbal symbols (Valdois et al., 2012). However, the VA span deficit disappears when dyslexics are asked to report the color of five dots in a row (Valdois et al., 2012) consistent with a specificity of VA impairment for stimuli made of separable features, as reported in visual search in the Casco and Prunetti (1996) study. Neuroimaging studies have identified the superior parietal lobule (SPL) bilaterally as the neural substrate of VA form of developmental dyslexia and the SPL as the key region for the simultaneous processing of multiple objects (Lobier et al., 2014, 2012a, 2012b; Peyrin et al., 2012, 2011; Reilhac et al., 2013). Thus, dyslexic people and patient IG, both with SPL dysfunction, show reduced attentional resource only for stimuli made by separable features (i.e. symbols). However, it is conceived as a reduced VA span (i.e. number of 
objects) in developmental dyslexia as tested with the Global Report task, and as a reduced VA space (i.e. attentional window) in simultanagnosia as tested with visual search tasks.

Patient IG was recently administered the Global Report task (Valdois et al., 2019). We observed that she showed a reduced VA span in terms of number of objects in the Global Report task, which stands in contrast with the results of visual search (Khan et al., 2016) in which patient IG exhibited a decrease of her space-based attentional window irrespective of the number of objects to process within this area at each fixation. Specifically, we observed that she was unable to process more than 3 consonants simultaneously, both in conditions of normal or wider spacing, while she was able to process 2 consonants or less similar to controls. Further, in a recent study, patient IG was unable to identify a target in peripheral vision when it was flanked by dissimilar distractors, but when the flankers were identical she could identify the central target (Vialatte et al., 2020). We hypothesized that this difference may have to do with differences in the tasks. In the Khan et al (2016) study, the visual search tasks comprised distractors that were all identical, reducing the visual uncertainty, whereas in the Global Report task measuring the VA span, letters were all different (Bosse et al., 2007; Valdois et al., 2019). We therefore argue that the fact that identical items can be grouped even with simultanagnosia (Luria, 1959) can explain why patient IG and healthy individuals displayed a spatially limited « working space » in visual search. Thus, we hypothesize that the use of a spatial attentional window versus an object-based span may depend on whether distractors are all identical or dissimilar.

Here we aimed at determining the factors underlying visual search attentional limitation in healthy individuals and in patient IG. We varied the stimulus type (objects made of separable features or not), the distractors (identical versus dissimilar) as well as set size. As in Khan et al. (2016), we used gaze-contingent visible windows of different diameters to measure the workspace used by healthy participants and patient IG for each set size. The logic behind using the gaze-contingent moving window paradigm is as follows: if participants perform search with a certain sized attentional window around fixation, then presenting a moving window larger than this attentional window should not increase search times, as participants would not have processed anything outside their attentional window in any case. However, if the moving window is smaller than the attentional window, search times would increase as the participant would have processed 
more objects within their attentional window and because of the smaller moving window, they are unable to do so. They thus require more eye movements to search the space, taking more time. Along these lines, the gaze-contingent window can also test for spacebased vs. object-based by looking at whether search times change in the same manner across set size for different moving windows (arguing for space-based) or whether they change differently (arguing for object-based). If visual search is based on fixed spatiallylimited attentional window, even for dissimilar distractors, this would correspond to a variable number of objects falling in a limited « working space » across set size. This search window will have to be displaced to cover the entire visual display size in which the distractors are homogenously distributed, which should be reflected by a mean number and mean amplitude of saccades performed to find the target depending on task difficulty but importantly not depending on set size. In contrast, if the group of stimuli processed simultaneously corresponds to a fixed number of objects, then search times and the mean number of saccades performed to find the target should instead increase and conversely saccade amplitudes should decrease with set size.

\section{METHODS}

\section{Participants}

Patient IG is a right-handed female who was 49 years old at the time of this experiment. She suffered from an ischemic stroke when she was 29. The resulting lesion involved all of area 7 and the intraparietal sulcus in both hemispheres, as well as the upper part of Brodmann's areas 19, 18 and 39 (corresponding to area PEG as defined by Eidelberg and Galaburda, 1984) but spared area 40 and the tempo-parietal junction (see Fig. 1a). Visual field perimetry showed a partial right inferior homonymous quadrantanopia with temporal crescent sparing (Fig. 1b), due to the subcortical damage of the optical radiations below the parietal cortex in the left hemisphere. Her binocular vision and visual acuity were normal (7/10 and 8/10 for the right and left eye respectively). She initially demonstrated a Balint syndrome (bilateral optic ataxia and simultanagnosia) without hemineglect. Her eye movements were completely normal during clinical assessment, with latencies and amplitudes similar to controls within the 
functional saccadic range: Gaveau et al. (2008) found deficits only for very far eccentricities, for example, $20^{\circ}$ or greater, saccade amplitudes which are almost never used in everyday life as they would normally be implemented with a head contribution. Simultanagnosia was so severe initially that it prevented her from perceiving two dots presented simultaneously, but this recovered quickly (Pisella et al., 2000). During the acute phase, she reported that she could not clearly see more than one finger of her hand at once; she is now able to see "almost" all fingers simultaneously. 10 years after her ischemic stroke (Khan et al., 2016), she could correctly distinguish all the objects in overlapping figures, but the largest figure was reported late and last. She also struggled more than control participants (she was successful but took more time) to determine the exit of simple visual maze. For the Navon test (Navon, 1977), she did not show the classical global precedence effect of healthy controls: she was not slower in identifying the local letter when it was incongruent with the global letter $(\mathrm{sH}, \mathrm{hS})$ compared with when it was congruent (sS, hH). 15 years after her ischemic stroke, IG was administered a general neuropsychological screening battery to explore the cognitive long-term effects of her brain damage (Valdois et al., 2019). She showed normal executive functions (as evaluated by the Wisconsin test), temporal attention shifting abilities (as evaluated by the attentional blink task and the stream segregation report), auditory attention and visual processing speed with single letters. Only on-line visuo-motor guidance and spatial visual attention were impaired. 


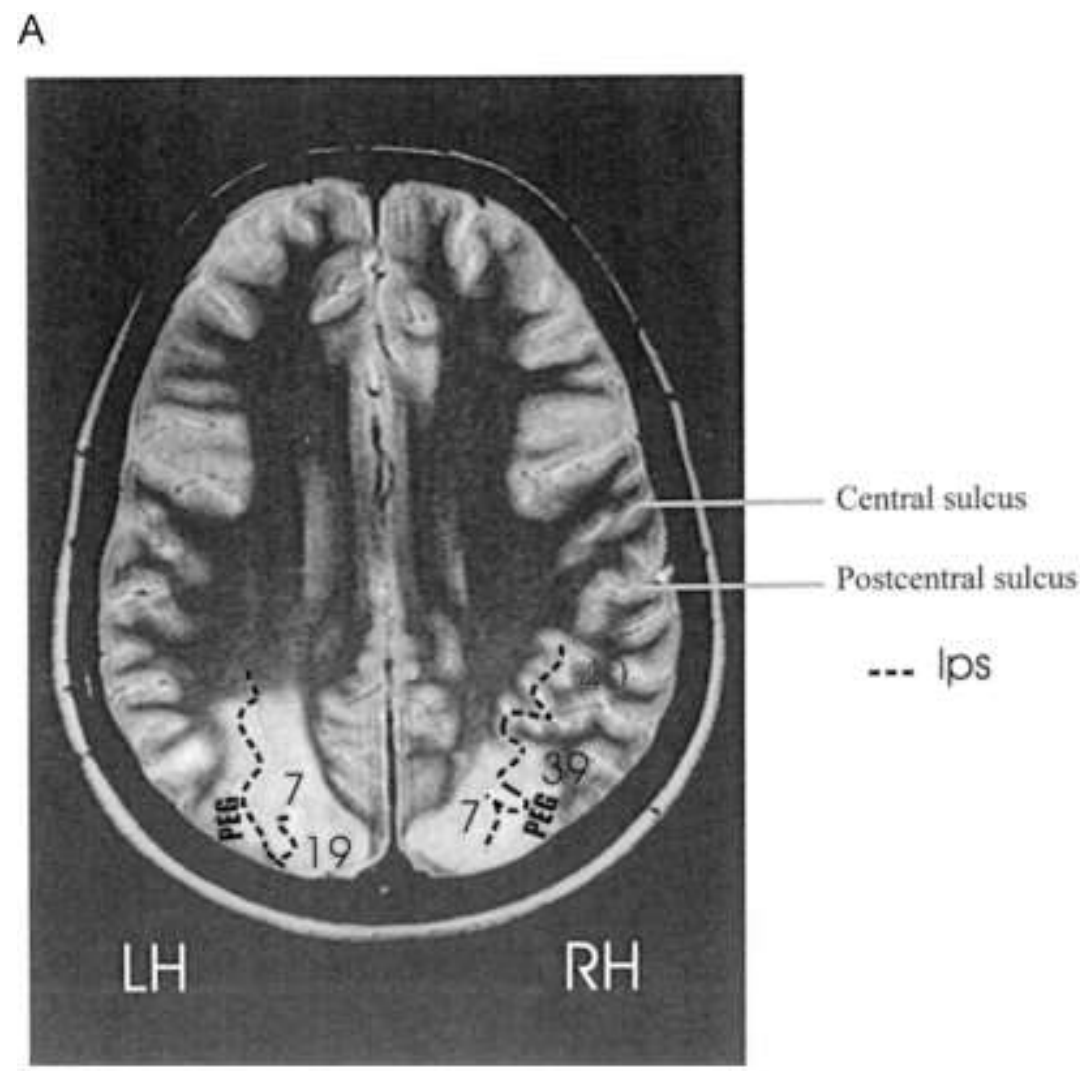

B

\section{Left Eye}

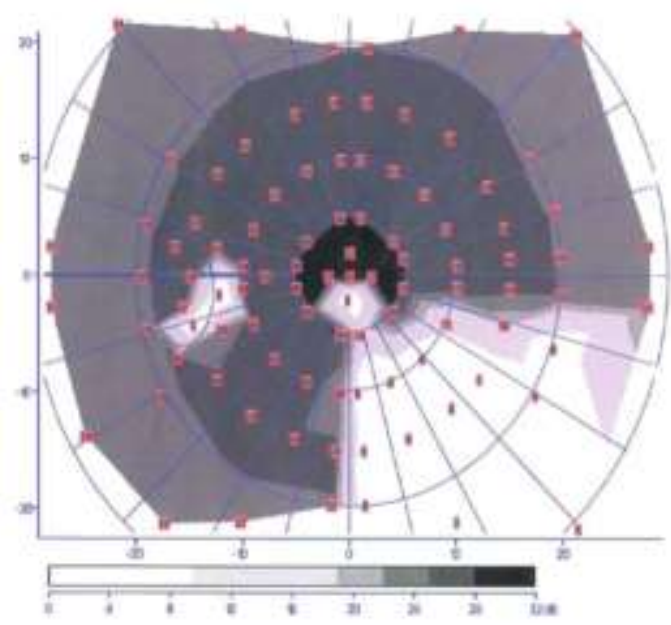

Right Eye

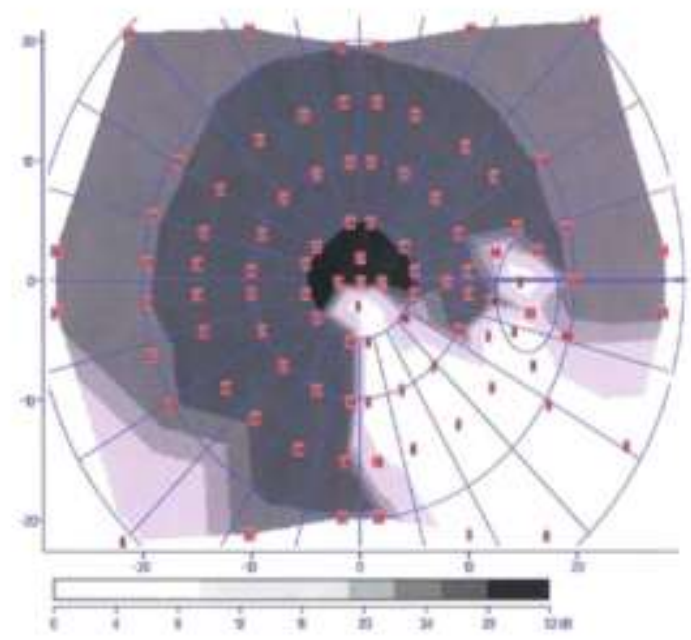

Figure 1 -. A-Horizontal section through IG's brain, visualized with structural MRI. B- Visual field perimetry for patient IG showing quadrantanopia in the right lower quadrant of the visual field of both eyes. 
In addition, different groups of neurologically intact controls participated in the different tasks. 6 control participants ( $M=38$ years, age range $=24-55$ years, 5 females) performed the 4 visual search tasks in full view and with a gaze-contingent quadrantanopic mask, 5 control participants $(M=30.4$ years, age range $=24-43$ years, 2 females) performed the color visual search tasks involving dissimilar distractors with several gaze-contingent visible windows of variable diameter and 6 control participants $(\mathrm{M}=35.5$ years, age range $=27-60$ years, 3 females $)$ performed the symbol visual search tasks involving dissimilar distractors with several gaze-contingent visible windows of variable diameter.

All experimental procedures were approved by the French health research ethics committee (CPP Nord-Ouest I, Lyon, 2017-A02562-51).

\section{Apparatus}

Participants sat in a semi-dark room with their eyes at a distance of $57 \mathrm{~cm}$ from a high-speed CRT monitor (dimensions: $40 \times 30 \mathrm{~cm}$, refresh rate: $160 \mathrm{~Hz}$ ), with their forehead and chin stabilized. Stimuli were presented on the screen using a real-time stimuli presentation (Visual Stimulus Generator ViSaGe, Cambridge Research System, Rochester, UK) along with custom-written code developed in the laboratory. Eye movements were recorded using a high-speed video Eyetracker (Cambridge Research System) at 1000 Hz. Participants responded using a ViSaGe response box.

\section{Stimuli and tasks}

The shape-color conjunction task (Fig 2A) consisted of finding a red disk among distractors that were red squares and green disks. The balloon feature-absent task (Fig 2B) consisted of finding a black circle (o) lacking a line among "balloons". These two tasks were the same as in Khan et al. (2016).

Two new tasks were designed to be similar but to prevent perceptual grouping of the distractors; they comprised dissimilar distractors which were all different. The color task (Fig 2C) consisted of finding the same red disk as the shape-color conjunction task but among disks of various colors. The symbol task (Fig 2D) consisted of finding the same black circle (o) as the balloon feature-absent task but among other black circles of variable sizes that were all combined with a line of different orientation and location. 
A - Conjunction Task

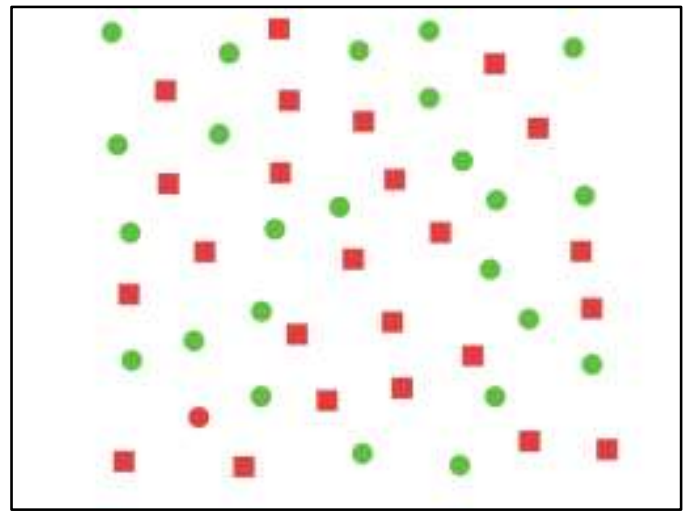

C- Color Task

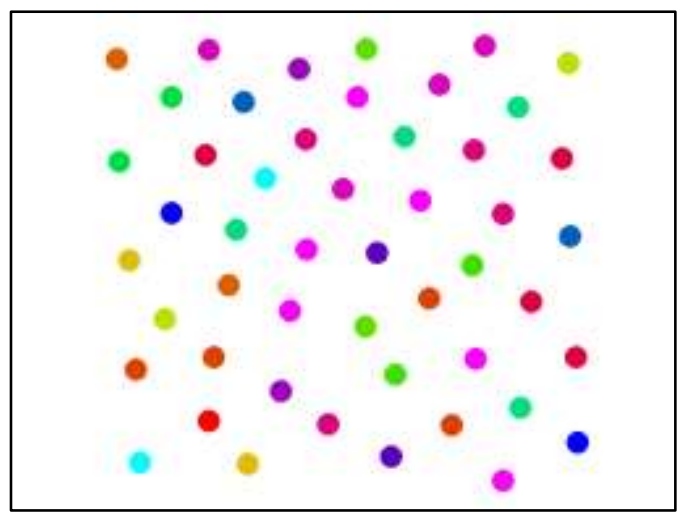

B- Balloon Task

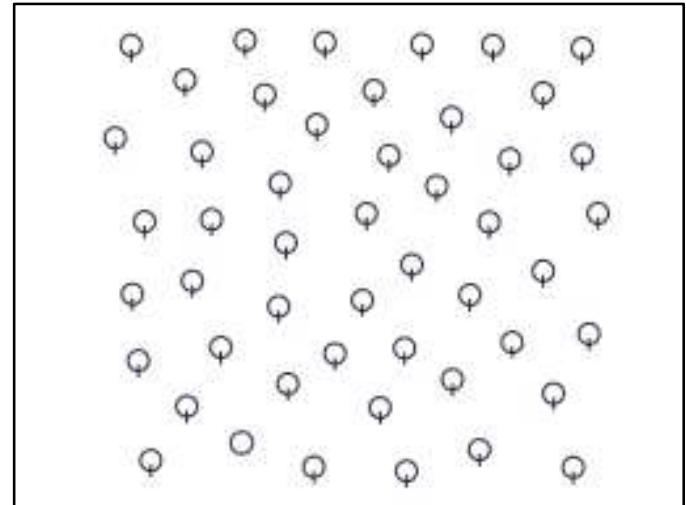

D- Symbol Task

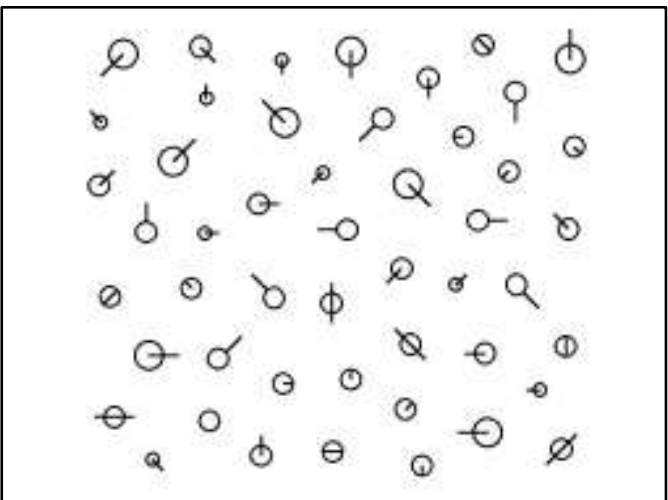

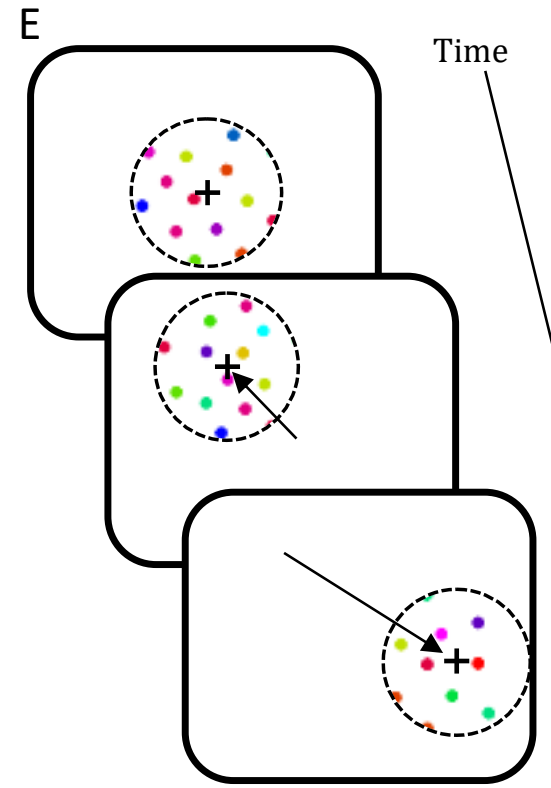

F

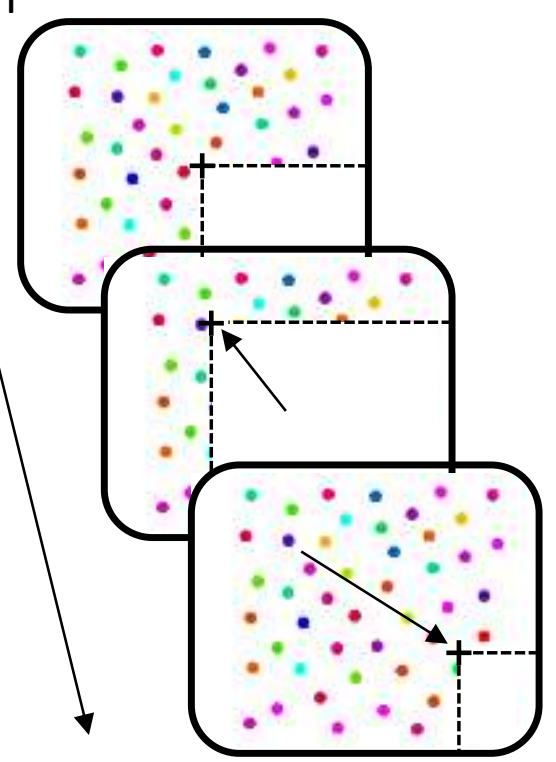

Figure 2 - The four visual search tasks (A: shape-color conjunction task, B: Balloon feature-absent task, C: Color task, D: Symbol task) illustrated for a set size of 49 and with the target in the lower left quadrant. E\&F: Schematization over time of what participants see when performing the color task with the two different gaze contingent displays (E: gaze-contingent visible window, F: quadrantanopic mask). Successive participant's gaze positions are indicated by a cross, saccades by an arrow. 
For each task, a trial began with a fixation dot at the center of the screen. Once fixation on the dot was detected, the dot was replaced by the stimulus array. Participants were asked to press the right button on a response box as fast as possible with their right hand if they detected a target among distractors (target-present trials) and press the left button with their left hand if there was no target in the stimulus array (target-absent trials).

Each block of trials consisted of 48 target-present trials (16 trials for each group of 12,24 , or 48 distractors, corresponding to set sizes of 13, 25 and 49 objects) and 6 targetabsent trials ( 2 trials of each set size) resulting in a total of 54 trials. The visual displays of different set-sizes, target eccentricities and quadrants were randomly intermingled within each block of trials with equal number of repetitions per condition. Within each random sequence of 48 target-present trials, the target $\left(1.3^{\circ}\right.$ diameter $)$ randomly appeared in one among the 4 quadrants, and randomly at an eccentricity of either $7.5^{\circ}$ or $12.5^{\circ}$ twice per quadrant. The distractors, depending on their number, were then distributed pseudo-randomly across the rest of the space with a minimum spacing of $1.3^{\circ}$ between objects. There was therefore increased spacing with smaller set sizes.

\section{Procedures}

Six control participants and patient IG performed the 4 visual search tasks in the full view condition. For each task, each control participant ran 1 block of trials that represented 216 trials in total (54 per visual search task) and patient IG ran 3 blocks for each task. These six control participants also performed one block each of the 4 tasks with a gaze-contingent quadrantanopic mask (Figure 2E right panel) to simulate the right lower quadrantanopia of the patient.

Six control participants ( 2 among them also performed the first part and the others were new control participants) performed the color and the symbol tasks with gazecontingent visible windows (Figure 2E left panel) of 12.5, 15, 20, 25 and $30^{\circ}$ diameter (one block each), in random order, to investigate the size and type of attentional field used in these new tasks. Controls who had not performed the first part before undertook the color and symbol tasks in the full view and quadrantanopic conditions. All additionally performed the symbol task with smaller moving windows of $6^{\circ}$ and $10^{\circ}$ diameter another day for the comparison to patient IG's performance. 


\section{Data analysis}

The total number of trials across the experiments for control participants was 6966 trials. Of those, 774 were target-absent trials (which were not analyzed) and 6192 were target-present trials. Patient IG performed 648 total trials, of which 576 were targetpresent trials. Target-absent trials represented $11 \%$ of total trials and were randomly presented among the target-present trials to deter participants from automatically pressing the target-present button. False-alarm errors (where the target was absent but was reported as present) represented $4 \%$ of the total target-absent trials for IG and $2.3 \%$ $(\mathrm{SD}=2.3)$ for control participants. Incorrect trials (omissions, where the target was present but was reported as absent) comprised $0.5 \%$ of all trials for IG and $1 \%(\mathrm{SD}=0.9)$ for control participants.

Only the correct target-present trials were analyzed. Search reaction times (RTs) were calculated for trials with correct button presses as the difference between the time of the stimulus array onset and of the button press. Mean RT were computed for each set size, participant and search task.

For the off-line ocular behavior analysis, the scanning saccade detection threshold was set at $500^{\circ} / \mathrm{s}$ and fixation was considered to have occurred if gaze remained within a spatial dispersion threshold of $1.5^{\circ}$ on both vertical and horizontal coordinates for at least $50 \mathrm{~ms}$. Saccades that began between 0 and $100 \mathrm{~ms}$ after the appearance of the stimulus array were considered anticipatory and were not included in the analysis. To take account of the decision time we did also not consider saccades that took place within $70 \mathrm{~ms}$ of the button press time. Mean number of saccades, mean saccade amplitude and mean fixation durations (time $>50 \mathrm{~ms}$ spent between consecutive saccades) were computed.

\section{Statistics}

For each visual search task performed in the full view condition, we evaluated the set size effect $(13,25$ or 49 objects) using one-way repeated-measures ANOVAs on the individual mean RTs, number of saccades, fixation durations and saccade amplitude. The cost-per-item (CPI) was calculated for each task using linear fits across the 3 set sizes. 
We also assessed the effect of gaze-contingent visible windows of increasing diameters $\left(12.5^{\circ}, 15^{\circ}, 20^{\circ}, 25^{\circ}\right.$ and $\left.30^{\circ}\right)$ in the two new visual search tasks (those with dissimilar distractors). A three-way repeated-measures ANOVA was first performed on the mean individual RTs with task, window size and set size as factors. Two-way repeatedmeasures ANOVAs were then performed to evaluate the effect of the different window sizes and set sizes for each task. T-tests fdr-corrected by task were performed for each set size between performance in full view and with each window diameter to determine the largest window for which RT was significantly increased.

At each set size, IG's performance was then compared to the control group performance with quadrantanopic mask for all 4 tasks, and to the control group performance with different moving windows in the symbol task, using Crawford modified t-tests (Crawford and Howell, 1998) fdr-corrected by task. 


\section{RESULTS}

$\underline{\text { Full view condition for control participants as a function of set size: }}$
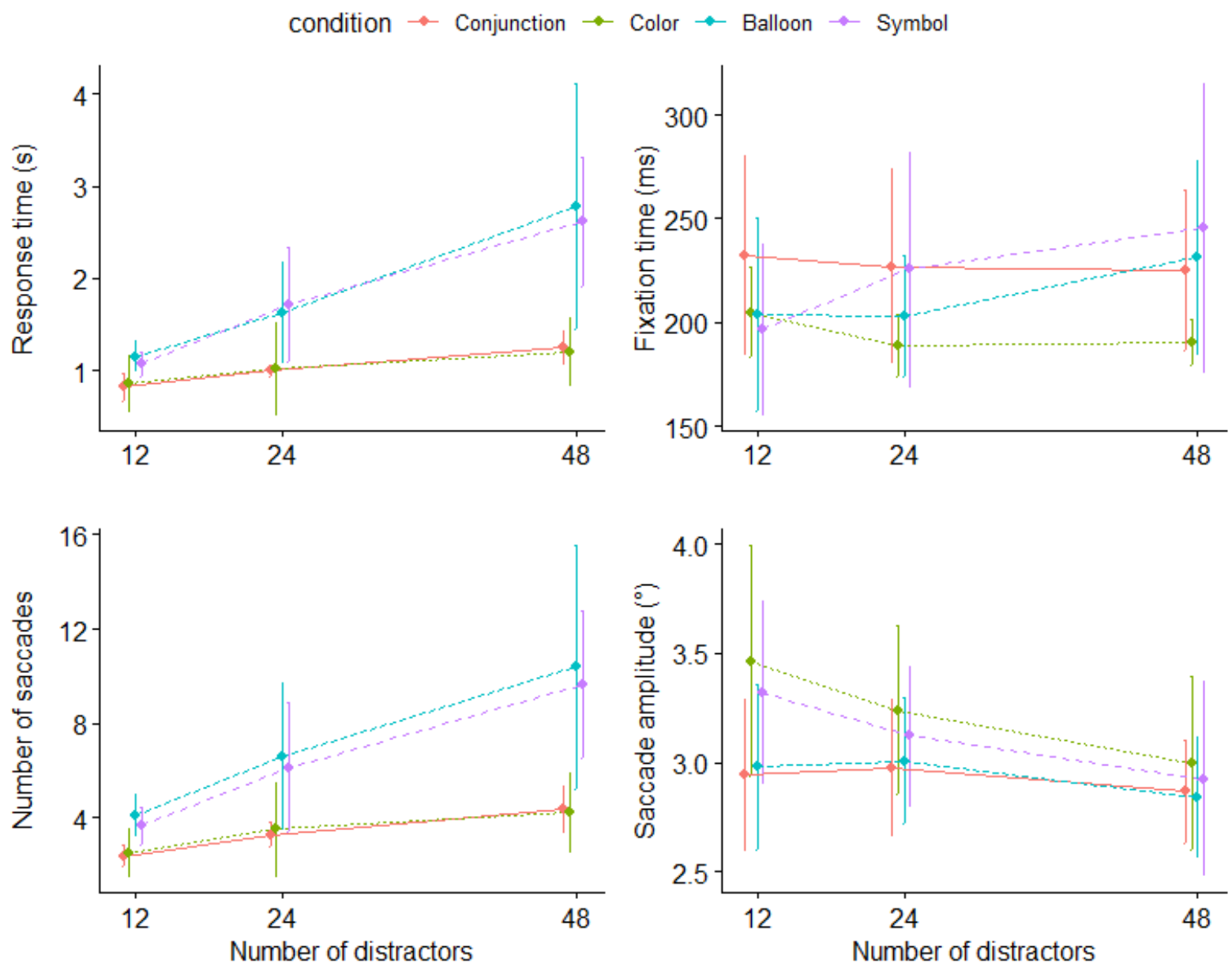

Figure 3 - Controls' mean performance (RT, fixation time, number and amplitude of saccades) in the different visual search tasks

We first investigated how control participants performed search in the four tasks, looking at reaction times, fixation times as well as number and amplitude of saccades.

$R T$

One-way ANOVAs performed separately for each task with set size as a factor showed a significant effect in all four tasks (all Fs $>16, \mathrm{p}<0.05$ ). As can be seen in Figure 3, RTs increased with increasing number of distractors. We calculated the cost-per-item for each task, as a linear fit across the three distractor numbers. The average cost-per-item was $9.5 \mathrm{~ms}(\mathrm{SD}=2.9)$ for the conjunction task, $9.2 \mathrm{~ms}(\mathrm{SD}=4.2)$ for the color task, $30.3 \mathrm{~ms}$ $(\mathrm{SD}=18.5)$ for the balloon task and $41 \mathrm{~ms}(\mathrm{SD}=14.8)$ for the symbol task. To summarize, 
control participants appear to perform the conjunction and color task in a similar manner, while the tasks involving stimuli made by separable features appear more "serial" (higher CPI), especially when distractors were all dissimilar.

\section{Fixation time}

One-way ANOVAs revealed a non-significant set size effect for all tasks (all Fs $<1.229$, p $>0.333$, Fig. 3).

\section{Number of saccades}

One-way ANOVAs showed a significant set size effect for all tasks (all Fs $>13.334$, $\mathrm{p}<0.05)$. As can be seen in Figure 3, the pattern was very similar to that of RT.

\section{Saccade amplitude}

One way ANOVAs showed a significant set size effect only for the symbol task $(F(2,10)=5.863, p<0.05)$; exploratory saccades were smaller for increasing set sizes (Fig 3D, purple line). For the other tasks, the set size had no significant effect on saccade amplitude (Fs<1.936, ps $>0.195)$.

To sum up, mean RTs and number of saccades increased with set size for all search tasks - a signature of a serial search process. The conjunction and balloon serial tasks were identical to those used by Khan et al. (2016) (conjunction task- CPI: 9.5ms vs. 6.4ms in Khan; balloon task - CPI: 30.3ms vs. 23 ms in Khan). Khan et al. (2016) tested different moving windows on these two tasks, while moving windows of $15^{\circ}$ diameter or less increased RT overall, they did not change the CPI (the slope of the RT function relative to set size), supporting the notion that they are performed with a similar space-based attentional workspace larger than $15^{\circ}$ but smaller than $20^{\circ}$ of diameter. To explain the higher increase of RT across set sizes in the balloon task (the higher CPI), fixation time could have been longer in this task and also longer for increasing set sizes, but this was not confirmed here. Only saccade number seemed to distinguish between these two visual search tasks of different CPI (with only the balloon task involving stimuli made by separable features). Then only saccade amplitude seemed to distinguish between the balloon and the symbol tasks (both involving separable feature stimuli but only the 
symbol task involving dissimilar distractors) and predict the space versus span process. Indeed, it varied significantly across number of distractors only for the symbol task.

\section{Effect of gaze-contingent visible windows of increasing diameters in the two new tasks} in controls:

A three-way repeated-measures ANOVA performed on RT as dependent variable revealed in the control group a main effect of task (color vs. symbol - $F(1,9)=10.15$, $p<0.05$ ), moving window diameter $(F(5,45)=50.63, p<0.05)$ and set size $(F(2,18)=37.43, p<0.05)$. There was a tendency for an interaction between these three factors $(F(10,90)=1.70, p=0.09)$ suggesting that in the symbol condition the cost of a given window depended on set-size, whereas it was constant across set-sizes in the color condition. Based on these results, we next analyzed the effect of moving window diameter and set size for the control group separately for the color and symbol.

\section{Color task performance for control participants}

A two-way repeated measures ANOVA revealed a main effect of moving window diameter $(\mathrm{F}(5,20)=49.83, \mathrm{p}<0.05)$ and of set size $(\mathrm{F}(2,8)=24.62, \mathrm{p}<0.05)$ on RT but no significant moving window by set size interaction effect $(F(10,40)=0.864, p=0.57)$. This can be seen in Figure 4, where RTs increase with decreasing moving windows but the slope remained similar across set size. If the attentional window size was object based, we would expect an interaction between the number of objects and the moving window diameter, because the cost in RT for a given moving window would vary depending on the number of objects within that space. On the other hand, if the attentional window is determined by spatial extent only, then the cost in RT for a certain sized visible window would be the same across the different number of objects. In sum, similar to the conjunction and balloon tasks (Khan et al., 2016), this color search also appears to be performed using a limited space-based attentional workspace.

To determine the size of this attentional workspace, we determined which moving window diameter resulted in a significant increase in RT compared to the full view condition (see introduction). Paired $\mathrm{t}$ - tests between RT in full view and the different moving window diameters revealed that while the moving window of $25^{\circ}(\mathrm{p}>0.05$, fdrcorrected) was not different from the full view condition, the $20^{\circ}$ moving window condition had significantly higher RTs $(\mathrm{p}<0.001)$. Therefore, we estimated that the control 
group's attentional window was larger than $20^{\circ}$ but smaller than $25^{\circ}$ in this search condition.

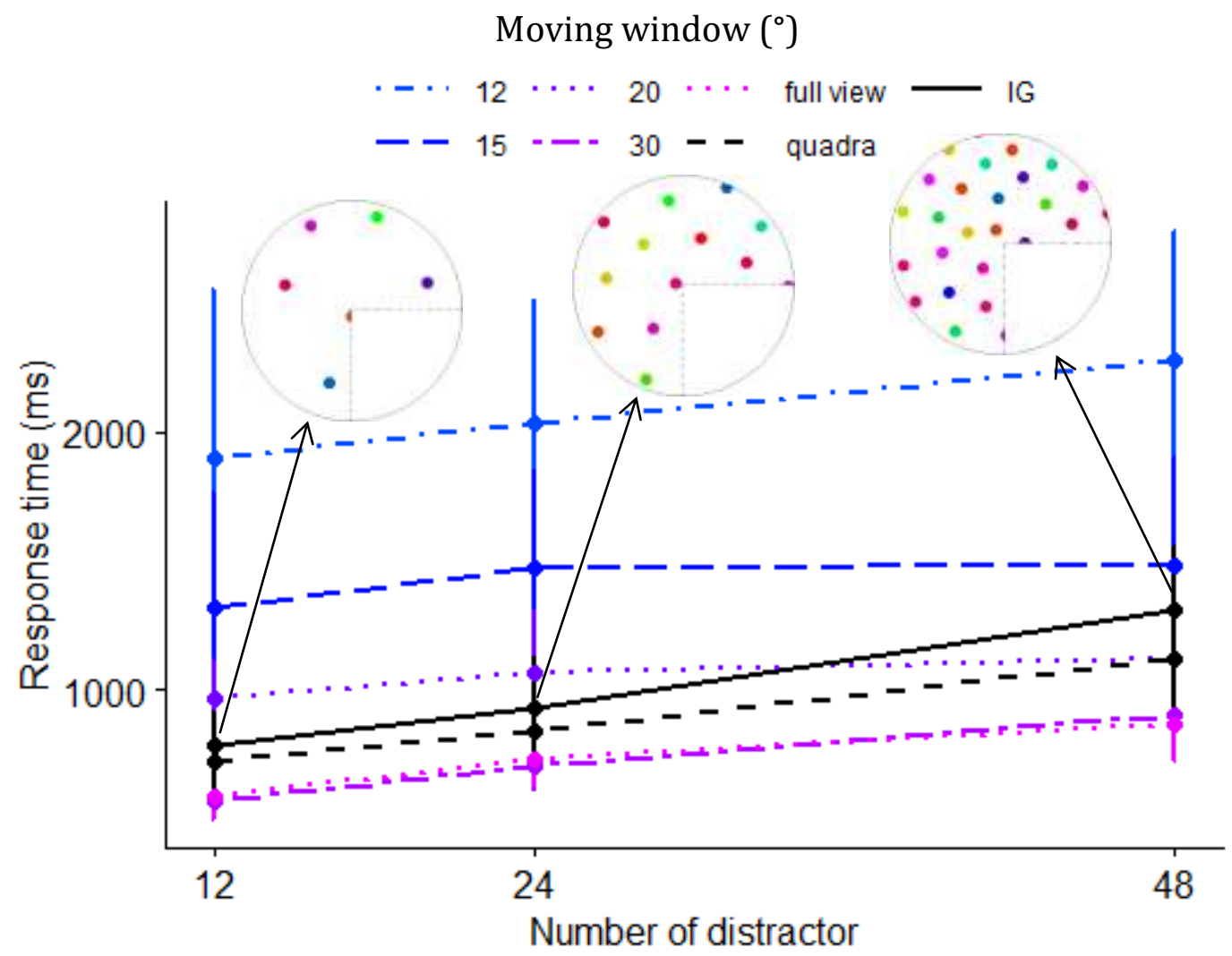

Figure 4: Color task - Effect of moving windows of increasing diameter on RT by set size in controls, compared with controls and patient IG RTs in the full view condition. Response time observed in control participants with a quadrantanopic mask simulating the patient's quadrantanopia is also illustrated because it was not significantly different from the patient's performance, which was also not significantly different from the controls' performance with a circular gaze-contingent visible window of $30^{\circ}$. The number of items likely processed at each fixation for each set size by the patient is therefore illustrated by schematic disks of $30^{\circ}$ diameter with a quadrantanopia.

\section{Symbol task performance for control participants}

For the symbol task, the two-way repeated measures ANOVA revealed a main effect of moving window diameter $(F(5,25)=21.27, \mathrm{p}<0.05)$, a main effect of set size $(F(2,10)=31.91, p<0.05)$ and also a significant interaction between these two factors $(F(10,50)=2.60, p<0.05)$. This significant interaction effect means that the different sized moving window cost in RT was not the same across the different set sizes: the cost of a given moving window (see for example $15^{\circ}$ on figure 5) was larger for smaller set size. Because of the interaction effect, we compared RTs across the different moving windows 
to the full view condition separately for each set size for controls using fdr-corrected paired t-tests. The smallest window that did not significantly increase RT compared to full view was $25^{\circ}$ for the set size of $12(\mathrm{t}=-2, \mathrm{p}=0.16), 20^{\circ}$ for the set size of $24(\mathrm{t}=-1.41$, $\mathrm{p}=0.32)$ and $15^{\circ}$ for the set size of $48(\mathrm{t}=-1.11, \mathrm{p}=0.41)$. This corresponded to $6-7$ items for the set size of $12,7-9$ items for the set size of 24 and 8-10 items for the set size of 48 . This similar number of objects processed across set sizes suggests that the symbol task was performed with a limited object-based attentional span rather than with a spacebased attentional window.

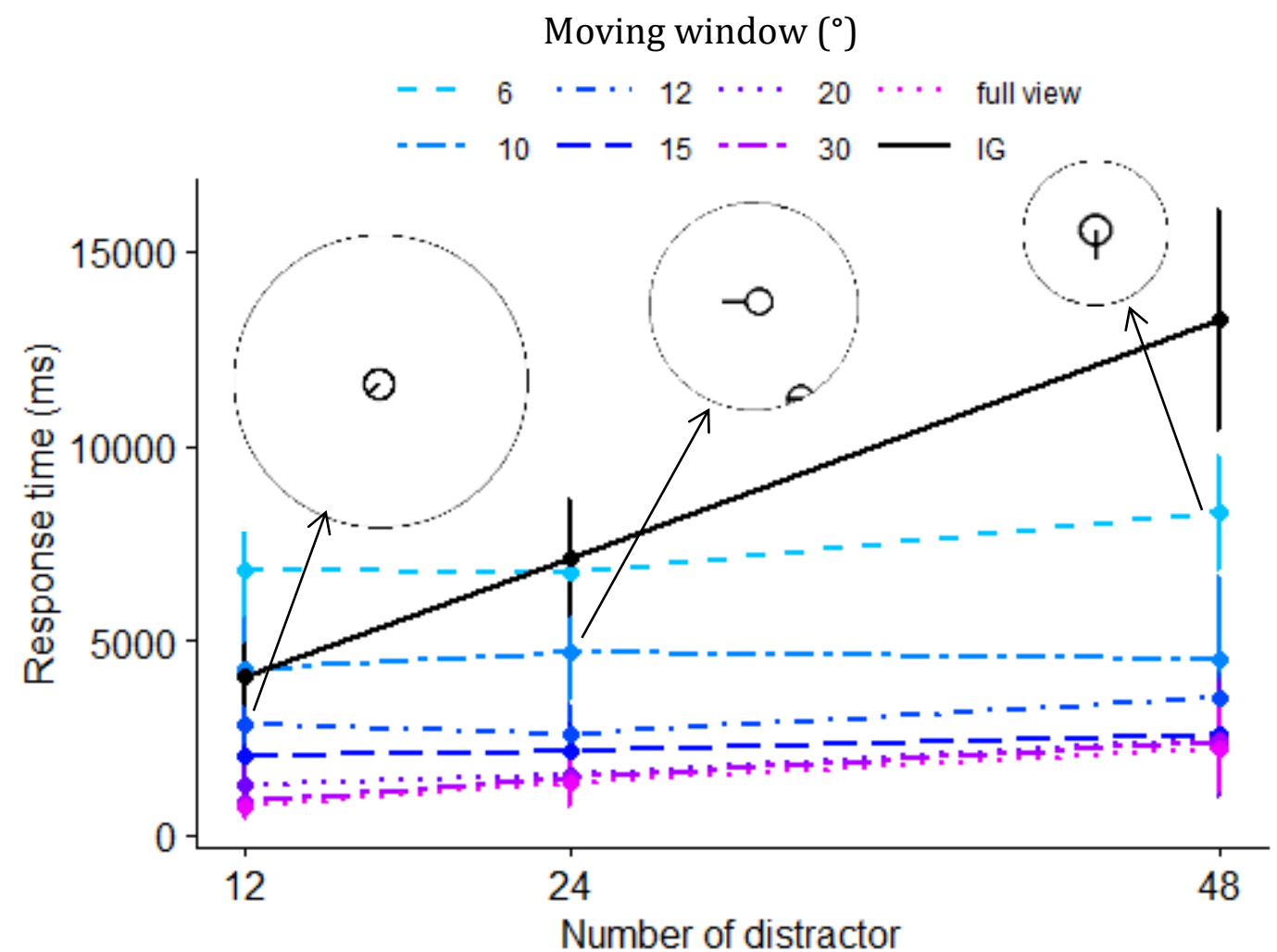

Figure 5 - Symbol task - Effect of moving windows of increasing diameter on RT by set size in controls, compared with controls and patient IG RTs in full view condition. No single moving window diameter was matching patient IG's performance. The moving window matching patient IG's RT in full view was $12^{\circ}$ for the set size of $12,10^{\circ}$ for the set size of 24 and under $6^{\circ}$ for the set size of 48. The number of items probably processed at each fixation for each set size by the patient is illustrated by schematic disks of corresponding decreasing diameter.

Comparison between patient IG's performance and control performance with gazecontingent quadrantanopic mask:

We first compared patient IG's performance (mean RTs, number of saccades and fixation durations) to that of the controls with a gaze-contingent mask simulating the right 
lower quadrantanopia of the patient using modified Crawford t-tests (Crawford and Howell, 1998). If patient IG's performance in a given visual search task was not different from controls' performance with the gaze-contingent quadrantanopic mask, then the patient was considered not having a high-level (visuo-attentional) deficit for the task. If this was not the case, we then compared IG's full view performance with controls' performance in the tasks with gaze-contingent visible windows of increasing diameters in order to determine whether the attentional deficit matched a fixed search field reduction.

$R T$

Patient IG was significantly slower than controls for all tasks (all $t>3.6, p<0.05$ ) except for the color task (all ts $<0.31$, ps $>0.05$ ) and for the smaller set size of conjunction $\operatorname{task}(t=2.1, p>0.05)$. As seen in Figure 6, for IG, the color task was the easiest even though it involved dissimilar distractors, followed by the conjunction task, and the RTs massively increased with set size for the tasks involving separable features, and even more so when distractors were dissimilar. This is reflecting in the cost-per-item for each task; it was 31.8 ms for the shape-color conjunction task, $14.8 \mathrm{~ms}$ for the color task, $125.4 \mathrm{~ms}$ for the balloon task and $253.8 \mathrm{~ms}$ for the symbol task.

Subject $\bullet$ Control subjects $\bullet-$ IG

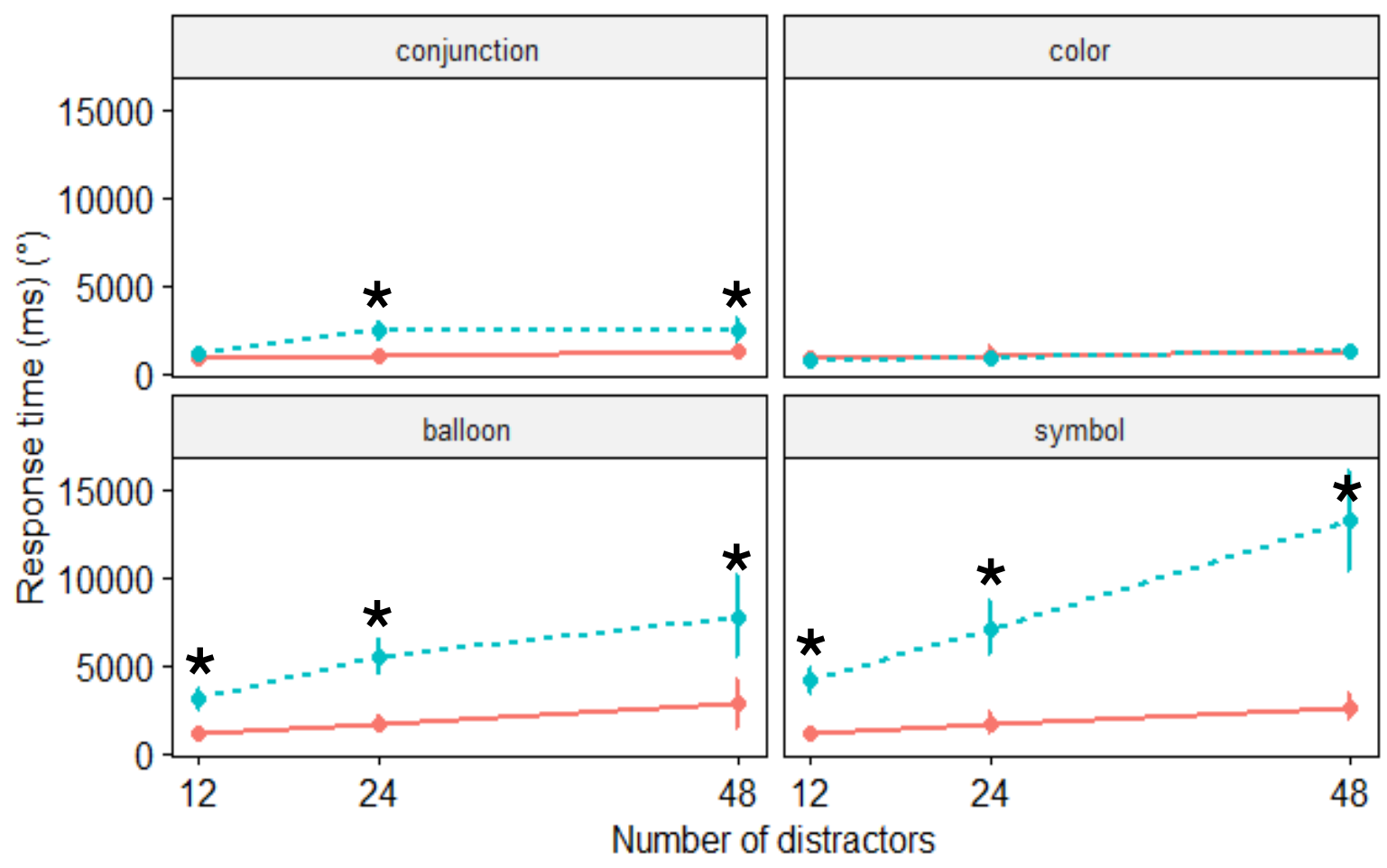


Figure 6: Response time (ms) for the 4 tasks, IG mean and inter-trials confidence interval in blue and control participants with the quadrantanopic mask mean and inter-individual confidence interval in red. ${ }^{*}=\mathrm{p}<0.05$.

\section{Fixation time}

Patient IG's fixation times were not different from controls except for the two biggest set sizes in the color task $(t>5.5, \mathrm{p}<0.05)$ (Figure 7$)$.

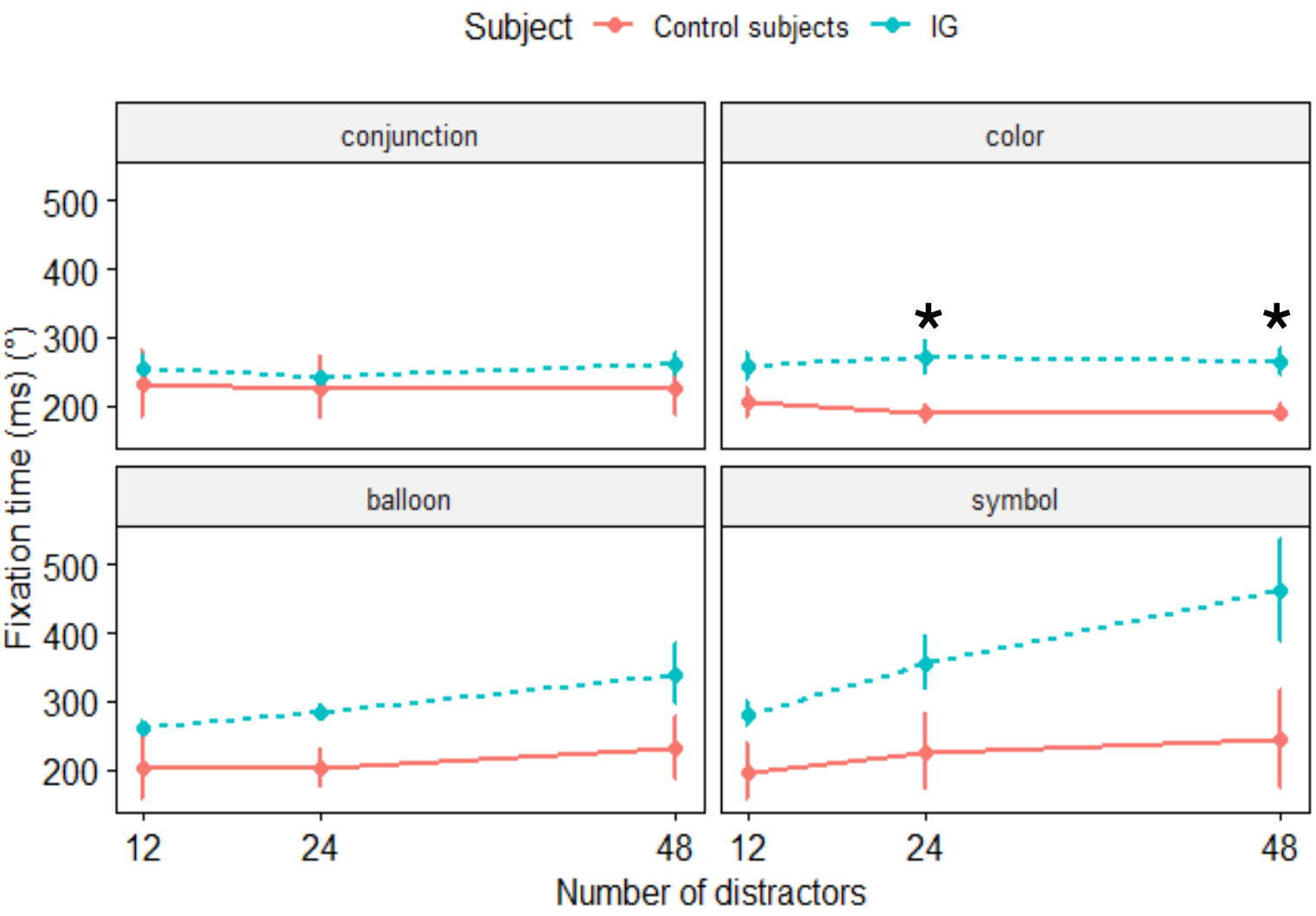

Figure 7 - Fixation time (ms) for the 4 tasks, IG mean and inter-trials confidence interval in blue and control participants with the quadrantanopic mask mean and inter-individual confidence interval in red. ${ }^{*}=\mathrm{p}<0.05$.

\section{Number of saccades}

IG made no more saccades than controls for the color task (all ts $<2.7$, ps $>0.05$ ). In the balloon task, she made more saccades but this was significant only for the smallest set size, while in the conjunction task she made more saccades for the two bigger set sizes. In the symbol task, she made more saccades for all three set sizes (all ts $>3.78$, ps $<0.05$ ) (Figure 8). 


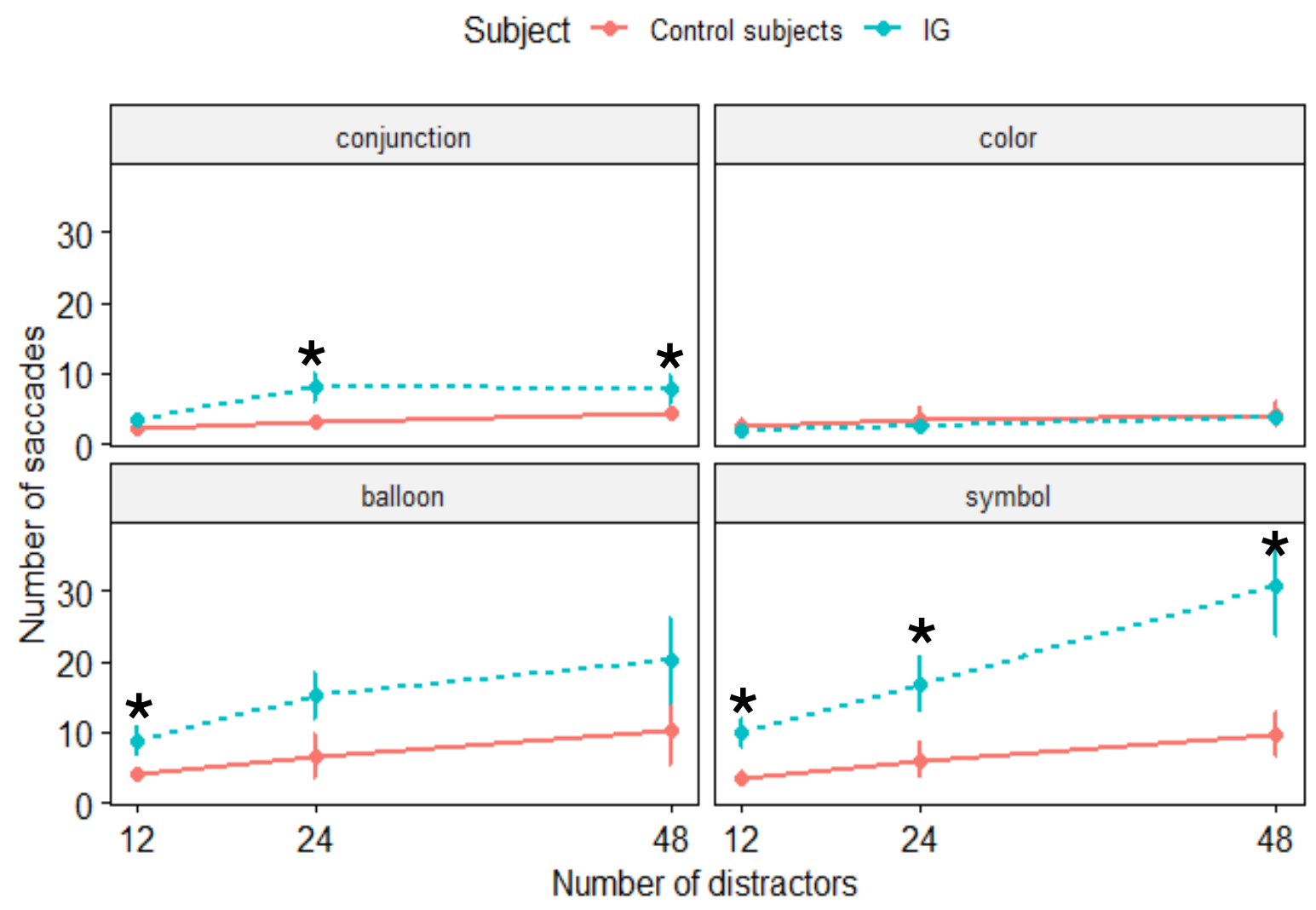

Figure 8 - Number of saccades for the 4 tasks, IG mean and inter-trials confidence interval in blue and control participants with the quadrantanopic mask mean and inter-individual confidence interval in red. ${ }^{*}=\mathrm{p}<0.05$.

\section{Saccade amplitude}

IG saccade amplitudes were not significantly different from controls with quadrantanopia for all tasks (all $\mathrm{t}<-2.3, \mathrm{p}>0.05$ ) except for the symbol task where $\mathrm{p}$ values equal to 0.05 were found at each set size and for the balloon task at set sizes 24 and 48 (Figure 9). 


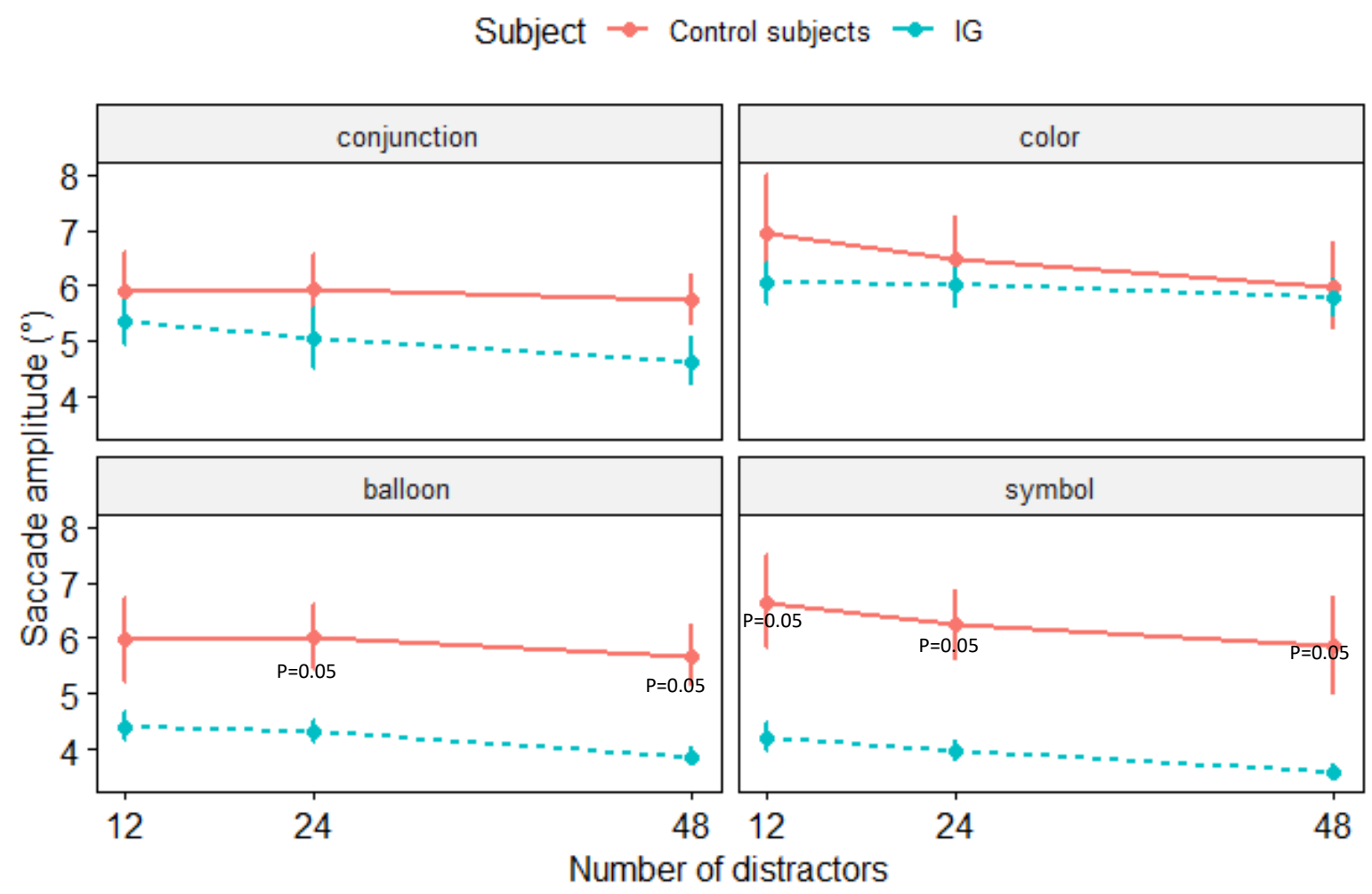

Figure 9 - Saccade amplitude $\left(^{\circ}\right.$ ) for the 4 tasks, IG mean and inter-trials confidence interval in blue and control participants with the quadrantanopic mask mean and inter-individual confidence interval in red. ${ }^{*}=p<0.05$.

To sum up, patient IG's RT was not different from controls with quadrantanopic mask in the color task, with nevertheless a higher fixation time observed in patient IG for the biggest set sizes. In the conjunction task, the slight increase of RT observed for the biggest set sizes in the present results for patient IG was reflected by a slight increase of the number of saccades. In the balloon and symbol tasks, the additional increase in RT was reflected by an increase of the number of saccades, and also by a decrease of saccade amplitude.

Comparison between IG's full view performance and controls' performance with different moving window diameters for the color and symbol tasks:

For the color task, the full view performance of patient IG was not different from controls' performance with quadrantanopic mask and the comparison between IG and control group with different moving windows using fdr-corrected Crawford tests showed that IG was not significantly slower than controls with the largest moving window tested $\left(30^{\circ}\right)$ at each set size. Based on these results, we conclude that IG was able, like control 
participants, to simultaneously process at each fixation a large area containing several objects (with non-separable features) which varied in number in this color task (figure 4).

For the symbol task, patient IG's RT was much higher than controls with quadrantanopic mask. Therefore, we compared her performance in full view at each set size with those of the control group with different moving window diameters, to determine the moving window where their performance at each set size was closest to patient IG's performance. This is similar to the comparison done in Khan et al. (2016) for the conjunction and balloon tasks. The moving window that best matched patient IG's RT in full view was $12.5^{\circ}$ for the set size of $12,10^{\circ}$ for the set size of 24 and under $6^{\circ}$ for the set size of 48 (corresponding to the largest control mask that not significantly different from IG's RT with fdr-corrected Crawford tests). The number of objects possibly falling within these moving windows corresponded to a fixed number of only 1 object: if an object was in the center of the window (fixated by IG) then no other object was visible in the window (figure 5). This scanning of the visual display object by object is in accordance with a number of saccades produced by patient IG on average in this task approaching the set size ( 9 for the set size of 12,15 for the set size of 24 and 28 for the set size of 48 , see figure 8). This averaged number of saccades is logically smaller than the set size because patient IG often found the target before having to explore the entire display. 


\section{DISCUSSION}

The tenets of the visuo-attentional (VA) theory of impaired reading performance argue that a subset of dyslexic children are unable process as many letters as normal reading children and therefore cannot develop any reading expertise. This VA impairment has been associated with SPL dysfunction (Lobier et al., 2014, 2012a, 2012b; Peyrin et al., 2012, 2011; Reilhac et al., 2013) and is viewed in the literature as a consequence of either a reduced VA span (Lobier and Valdois, 2015; Valdois et al., 2019) or a reduced "spotlight" (Khan et al., 2016; Vidyasagar, 1999; Vidyasagar and Pammer, 2010). Here we tested several visual search tasks in control participants and in a patient with SPL dysfunction in order to determine whether their VA limitation was a span (i.e. a limited number of items as in working memory) or a space (or attentional field i.e. a spatially limited focus of attention around gaze fixation corresponding to the metaphor of the "spotlight").

In the color task, the group of items processed in parallel at each fixation in control participants corresponded to a fixed spatial area in which a variable number of items could lie depending on set size; the effect of reduced moving windows did not interact with set size. Like controls, patient IG was able to process several items simultaneously at each fixation, even though the color task involved dissimilar distractors that could not be perceptually "grouped". Such a spatially limited attentional field was also previously demonstrated both for controls and for patient IG in the balloon task, a task involving symbols (objects made by separable features) in a condition of identical distractors (Khan et al., 2016). In the balloon task however, IG performed the task with a reduced spatial area, as replicated by the present results. These results confirm her impairment in integrating multiple separable visual features and her relatively preserved ability to process multiple objects made with non-separable features (Khan et al., 2016; Vialatte et al., 2020), whether distractors are identical or not. With identical distractors, this impairment is reflected by a reduction in the size of the spatial attentional window compared to when the visual search is performed by controls.

In contrast, for the symbol task in which distractors are all dissimilar, we found significant interaction effect of window sizes and set size on reaction time indicating a search process based on object number in healthy controls (estimated to be 6-7 items at 12, 5-6 items at 24 and 8-10 items at 48). Again, Patient IG's performance in this task 
involving separable features and dissimilar distractors did not differ in the nature of the search process (also object based) but was simply reduced compared to controls. Patient IG performance corresponded to the use of a span limited to one single object.

Patient IG's cost per item was longer than $250 \mathrm{~ms}$ in this symbol task involving distractors comprising only two separable features but with different orientations and relative positions. As a comparison, her CPI was $220 \mathrm{~ms}$ in the feature-absent character task of the study of Khan et al. (2016) in which all stimuli were "chinese-like" characters combining 4 (for the target) and 5 (for the distractors, all identical) separable features. Hence the symbol task appears to be more difficult because of its visual uncertainty due to dissimilar distractors that cannot be visually "grouped". This uncertainty seems to have a larger impact than having to visually integrate 5 separable features for each item.

One can also speculate that the switch between space and object-based span limitation observed in control participants between the balloon and the symbol task is due to the fact that when distractors are all dissimilar they cannot be perceptually grouped to alleviate crowding. Indeed, the objects are closer to each other when set size increases, so maybe the participants could not identify the target among the other objects lying at the extremity of their (fixed) attentional field due to crowding. This would also explain that a task can be serial (i.e. RT increases significantly with set size) even though 1) RT increases with moving window reduction without interacting with set size, 2) saccade amplitude does not significantly decrease with set size and 3) the fixation duration does not significantly increase with set size (as previously shown by Young and Hulleman (2013) and confirmed by the present results). The only saccade parameter that depends on set size and reflects RT is the number of saccades. Even if a fixed spatial window of search is used irrespective of set size, the items lying at the periphery of this attentional window may be crowded when set size increases leading the participants to revisit this spatial area several times with a slightly different gaze position to check again the presence of the target.

To sum up, we found that 1) when control participants use a fixed spatial attentional window, patient IG displays a similar behavior but with a smaller spatial area if stimuli are made by separable features, and that 2) when control participants turn to a different visual search process with an object span limitation probably due to crowding of dissimilar symbols, patient IG in turn displays an extremely reduced span, processing objects one by one. This drastic deficit of simultaneous perception consecutive to SPL 
dysfunction was observed only in condition overloading the variety of separable features locations to integrate (as in the symbol task of the present study). Interestingly, reading for a beginner is such a condition that implies the simultaneous perception of numerous dissimilar letters made by multiple separable lines and should crucially require SPL contribution. Accordingly, Moulton et al (2019) have shown that the connectivity between the parietal lobe and the occipito-temporal region that will become the visual word form area (VWFA) improves when children learn to read. Moreover, this myelinization of the parietal-to-VWFA territory connections correlate with improvements in reading scores over the first year of instruction.

\section{Acknowledgements:}

The authors want to thank patient IG and the age-matched controls for their kind participation to this study funded by Centre National de la Recherche Scientifique (CNRS) and Labex/Idex ANR11-LABX-0042 (France; grants to L.P.); Fondation de France, Fondation Mustela and Pulsalys (grants to A.V.). 
Bálint, R., 1909. Seelenlähmung des "Schauens", optische Ataxie, räumliche Störung der Aufmerksamkeit. ENE 25, 67-81. https://doi.org/10.1159/000210465

Bogon, J., Finke, K., Schulte-Körne, G., Müller, H.J., Schneider, W.X., Stenneken, P., 2014. Parameter-based assessment of disturbed and intact components of visual attention in children with developmental dyslexia. Dev Sci 17, 697-713. https://doi.org/10.1111/desc. 12150

Bosse, M.-L., Tainturier, M.J., Valdois, S., 2007. Developmental dyslexia: the visual attention span deficit hypothesis. Cognition 104, 198-230. https://doi.org/10.1016/j.cognition.2006.05.009

Casco, C., Prunetti, E., 1996. Visual search of good and poor readers: effects with targets having single and combined features. Percept Mot Skills 82, 1155-1167. https://doi.org/10.2466/pms.1996.82.3c.1155

Crawford, J.R., Howell, D.C., 1998. Comparing an Individual's Test Score Against Norms Derived from Small Samples. The Clinical Neuropsychologist 12, 482-486. https://doi.org/10.1076/clin.12.4.482.7241

Eidelberg, D., Galaburda, A.M., 1984. Inferior parietal lobule. Divergent architectonic asymmetries in the human brain. Arch Neurol 41, 843-852. https://doi.org/10.1001/archneur.1984.04050190049013

Gaveau, V., Pélisson, D., Blangero, A., Urquizar, C., Prablanc, C., Vighetto, A., Pisella, L., 2008. Saccade control and eye-hand coordination in optic ataxia. Neuropsychologia 46, 475-486. https://doi.org/10.1016/j.neuropsychologia.2007.08.028

Germano, G.D., Reilhac, C., Capellini, S.A., Valdois, S., 2014. The phonological and visual basis of developmental dyslexia in Brazilian Portuguese reading children. Front Psychol 5, 1169. https://doi.org/10.3389/fpsyg.2014.01169

Husain, M., Stein, J., 1988. Rezsö Bálint and his most celebrated case. Arch Neurol 45, 8993. https://doi.org/10.1001/archneur.1988.00520250095029

Khan, A.Z., Prost-Lefebvre, M., Salemme, R., Blohm, G., Rossetti, Y., Tilikete, C., Pisella, L., 2016. The Attentional Fields of Visual Search in Simultanagnosia and Healthy Individuals: How Object and Space Attention Interact. Cereb. Cortex 26, 12421254. https://doi.org/10.1093/cercor/bhv059

Lobier, M., Dubois, M., Valdois, S., 2013. The role of visual processing speed in reading speed development. PLoS One 8, e58097. https://doi.org/10.1371/journal.pone.0058097

Lobier, M., Peyrin, C., Le Bas, J.-F., Valdois, S., 2012b. Pre-orthographic character string processing and parietal cortex: a role for visual attention in reading? Neuropsychologia 50, 2195-2204. https://doi.org/10.1016/j.neuropsychologia.2012.05.023

Lobier, M., Peyrin, C., Pichat, C., Le Bas, J.-F., Valdois, S., 2014. Visual processing of multiple elements in the dyslexic brain: evidence for a superior parietal dysfunction. Front Hum Neurosci 8, 479. https://doi.org/10.3389/fnhum.2014.00479

Lobier, M., Valdois, S., 2015. Visual attention deficits in developmental dyslexia cannot be ascribed solely to poor reading experience. Nat Rev Neurosci 16, 225. https://doi.org/10.1038/nrn3836-c1 
Lobier, M., Zoubrinetzky, R., Valdois, S., 2012a. The visual attention span deficit in dyslexia is visual and not verbal. Cortex 48, 768-773. https://doi.org/10.1016/j.cortex.2011.09.003

Luria, A.R., 1959. Disorders of "simultaneous perception" in a case of bilateral occipitoparietal brain injury. Brain 82, 437-449. https://doi.org/10.1093/brain/82.3.437

Michel, F., Henaff, M.-A., 2004. Seeing without the occipito-parietal cortex: Simultagnosia as a shrinkage of the attentional visual field. Behav Neurol 15, 3-13. https://doi.org/10.1155/2004/836830

Moulton, E., Bouhali, F., Monzalvo, K., Poupon, C., Zhang, H., Dehaene, S., DehaeneLambertz, G., Dubois, J., 2019. Connectivity between the visual word form area and the parietal lobe improves after the first year of reading instruction: a longitudinal MRI study in children. Brain Struct Funct. https://doi.org/10.1007/s00429-019-01855-3

Navon, D., 1977. Forest before trees: The precedence of global features in visual perception. Cognitive Psychology 9, 353-383. https://doi.org/10.1016/00100285(77)90012-3

Peyrin, C., Démonet, J.F., N'Guyen-Morel, M.A., Le Bas, J.F., Valdois, S., 2011. Superior parietal lobule dysfunction in a homogeneous group of dyslexic children with a visual attention span disorder. Brain Lang 118, 128-138. https://doi.org/10.1016/j.bandl.2010.06.005

Peyrin, C., Lallier, M., Démonet, J.F., Pernet, C., Baciu, M., Le Bas, J.F., Valdois, S., 2012. Neural dissociation of phonological and visual attention span disorders in developmental dyslexia: FMRI evidence from two case reports. Brain Lang 120, 381-394. https://doi.org/10.1016/j.bandl.2011.12.015

Pisella, L., Gréa, H., Tilikete, C., Vighetto, A., Desmurget, M., Rode, G., Boisson, D., Rossetti, Y., 2000. An "automatic pilot" for the hand in human posterior parietal cortex: toward reinterpreting optic ataxia. Nat Neurosci 3, 729-736. https://doi.org/10.1038/76694

Reilhac, C., Peyrin, C., Démonet, J.-F., Valdois, S., 2013. Role of the superior parietal lobules in letter-identity processing within strings: FMRI evidence from skilled and dyslexic readers. Neuropsychologia 51, 601-612. https://doi.org/10.1016/j.neuropsychologia.2012.12.010

Valdois, Lassus-Sangosse, D., Lallier, M., Moreaud, O., Pisella, L., 2019. What bilateral damage of the superior parietal lobes tells us about visual attention disorders in developmental dyslexia. Neuropsychologia 130, 78-91. https://doi.org/10.1016/j.neuropsychologia.2018.08.001

Valdois, S., Lassus-Sangosse, D., Lobier, M., 2012. Impaired letter-string processing in developmental dyslexia: what visual-to-phonology code mapping disorder? Dyslexia 18, 77-93. https://doi.org/10.1002/dys.1437

Vialatte, A., Yeshurun, Y., Khan, A.Z., Rosenholtz, R., Pisella, L., 2020. Superior Parietal Lobule: A Role in Relative Localization of Multiple Different Elements. Cereb Cortex. https://doi.org/10.1093/cercor/bhaa250

Vidyasagar, T.R., 1999. A neuronal model of attentional spotlight: parietal guiding the temporal. Brain Research Reviews 30, 66-76. https://doi.org/10.1016/S01650173(99)00005-3

Vidyasagar, T.R., Pammer, K., 2010. Dyslexia: a deficit in visuo-spatial attention, not in phonological processing. Trends Cogn Sci 14, 57-63. https://doi.org/10.1016/j.tics.2009.12.003 
Wolpert, I., 1924. Die simultanagnosie — störung der gesamtauffassung. Z. f. d. g. Neur. u. Psych. 93, 397-415. https://doi.org/10.1007/BF02900065

Young, A.H., Hulleman, J., 2013. Eye movements reveal how task difficulty moulds visual search. J Exp Psychol Hum Percept Perform 39, 168-190. https://doi.org/10.1037/a0028679

Zoubrinetzky, R., Bielle, F., Valdois, S., 2014. New insights on developmental dyslexia subtypes: heterogeneity of mixed reading profiles. PLoS ONE 9, e99337. https://doi.org/10.1371/journal.pone.0099337

Zoubrinetzky, R., Collet, G., Serniclaes, W., Nguyen-Morel, M.-A., Valdois, S., 2016. Relationships between Categorical Perception of Phonemes, Phoneme Awareness, and Visual Attention Span in Developmental Dyslexia. PLoS One 11, e0151015. https://doi.org/10.1371/journal.pone.0151015 